\title{
Thermal Detection System for Not Superficial Victims Heartbeat Detection
}

\author{
Carlos I. García S., A. Yoloxóchil J. R., and Larry H. Escobar
}

\begin{abstract}
This article proposes a system for detecting people who have been trapped under rubble and remain in inaccessible confined spaces. The system is based on thermal imaging processing. It applies algorithms to search not superficial victims focused on detecting their heartbeat, so a module portable search installed in an exploration robot as well as an external module that processes all information transmitted using the Robot Operation System (ROS) are implemented.
\end{abstract}

Index Terms-Heartbeat, infrared radiation, IR, Mexican hat, region of interest, ROI, robot operating system, ROS, thermal images.

\section{INTRODUCTION}

Detecting victims trapped under rubble is usually done with the help of search dogs or auxiliary electronic equipment. Both forms of search are conducted from the surface, requiring certain conditions to be implemented, for example, in the case of dogs, they work for short periods and take breaks, both of the same length of time; acoustic detection systems to find calls for help, requires the search area as quiet as possible; video equipment requires light to provide an image, among others.

Most of these assistive technologies, despite implementing complex and robust algorithms for automatic detection of search variables, operate without the detection of vital signs, we think this knowledge could optimize the rescue and care for victims, and in addition, the range of scope of these technologies is limited.

The first response to such emergencies is provided by witnesses of the event. They help injured or trapped people at surface. Then, the search crews assess the area and organize activities to carry out according to international protocols, with the goal of finding survivors in security areas or under debris. The problem is the time spent in this planning, which can be reduced with assistive technology.

To explore and search survivors in dangerous and/or difficult access areas, it has been proposed the use of robots that are able to move on and through the rubble. These robots must port detectors and sensors that help to detect victims, especially those not superficial. For example, the snake robot Souryu IV, product of the team led by Dr. Satoshi Tadokoro

Manuscript received on April 25, 2016; revised September 20, 2016. This work was supported by the Digital Signal Processing Laboratory at the Postgraduate Engineering School of the National Autonomous University of Mexico.

The authors are with the Faculty of Engineering at the National Autonomous University of Mexico, Coyoacán, Mexico City, 04510, Mexico (e-mail: karlos.kigs@gmail.com, a_yolo@yahoo.com.mx, larryesc@gmail.com).
[1], allows scanning with its 32 video cameras the space that the robot is moving through, hoping finding any survivor in its exploration.

While robots are being developed, some people analyze, study and propose systems to detect victims more effectively, robust to environmental conditions in which they work and aimed at identifying vital signs remotely, such as cardiac pulse victims, breathing rate, and temperature among others. One of this proposal is to implement radar UWB dedicated to detecting heartbeat and/or breathing frequency. This technology is being developed by many working groups from universities, institutes and companies, as the project Finding Individuals for Disaster and Emergency Response (FINDER) of NASA, or the radar Leader Scan of the company LEADER, whose results depend on the properties of the medium in which electromagnetic waves travel.

However, the application of thermal imaging processing and conventional video has allowed the characterization and simulations of the heart rate at distance, as the work of Chekmenev [2] and other researchers able to detect and bring the beats per minute of a person, observing and analyzing the pattern of thermal infrared radiation characteristic of the carotid artery due to bloodstream.

Moreover Guha [3] estimated the heartbeat by studying certain parts of the face in images of conventional video based on the slight movement of certain areas of the face due to blood circulation, however the results proved dependency on factors inherent to the processing (noise, lighting variations).

Working with images outside the visible range, you will have advantages and disadvantages, the bandwidth in which they work, as Jin Fei and Ioannis Pavlidis [4] who used a thermal camera middle wavelength (MWIR) characterized the signal of human breath away due to temperature variations present when inhaling and exhaling into the nostrils.

Our interest in this topic is to reduce the time which involves organizing different response tasks, with the aim of rescuing and caring for not superficial victims. We propose the design of a portable victim detection system to be installed in mobile robots, based mainly in the processing of thermal imaging to detect the heartbeat and later incorporate other useful elements for detecting not superficial victims.

This article describes the detection system proposed, its implementation, the search methodology and the first results in terms of pulse detection.

\section{PRoposed DETECTION SYSTEM}

In its first stage, the detection system consists mainly of a thermal camera, which captures the infrared radiation (IR) in a defined range of wavelengths, according to type of detector 
equipment.

To select a model for our system we consider to determine the range of wavelength where most infrared thermal radiation takes place due to the average temperature of a person, $36.6^{\circ} \mathrm{C}$. By applying the Planck radiation law considering that the emissivity of the human body is 0.98 [5] we obtained the wavelength range more suitable for the detection of people, according to Fig. 1, we can observed much energy within the range of 7 to $12 \mu \mathrm{m}$, being the maximum point $9.37 \mu \mathrm{m}$ according to the Stefan-Boltzmann law. Therefore, the camera detector must be sensitive to the window of long wavelengths (LWIR).

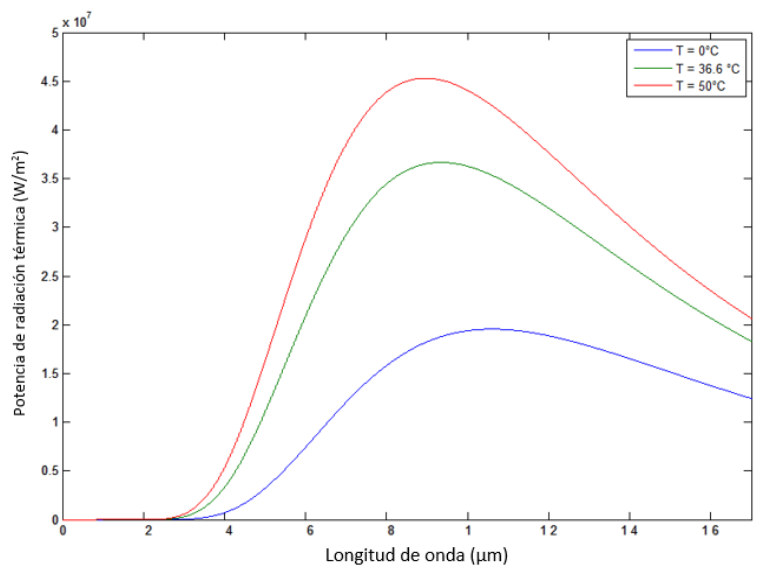

Fig. 1. Graph of the radiation spectrum according to human body temperature

Heat variations in the arteries and veins of the body human are below $100 \mathrm{mk}$ [6], so the minimum sensitivity of commercial equipment must be of $80 \mathrm{mk}$, even, they tend to be more sensitive. We chose the FLIR A35 camera considering the mentioned data related to thermal cameras due to its characteristics which satisfy the requirements.

\section{A. Detection System Architecture}

The proposed system detection is divided into two; the search module (portable unit, installed in the robot) which consists of an embedded system or mini-computer who acquires the video and retransmit to processing module. It's responsible for implementing the people search algorithm. And, the other is pulse detection. The Fig. 2 shows the structure of the system.

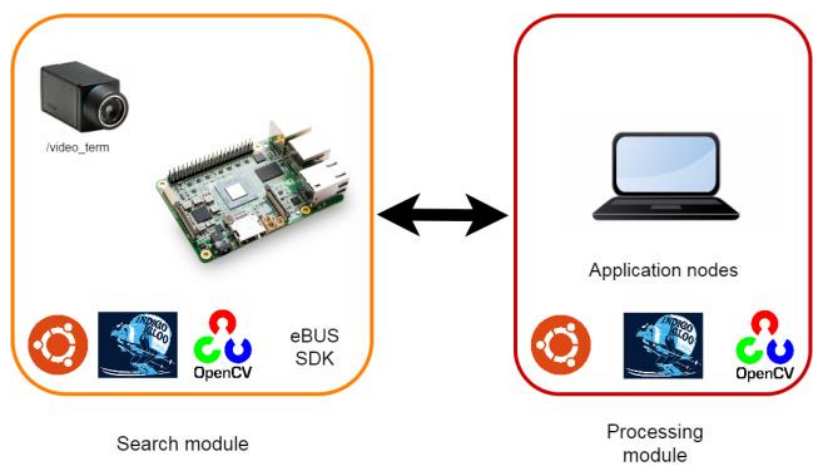

Fig. 2. Detection system structure.

The exchange of information between system modules is performed by ROS Indigo [7], creating a local network running the ROS Master in the processing station. One of the advantages of using this communication interface is that it eliminates the need to configure the camera with the eBUS SDK in case multiple users require access to the video transmission, however they should be connected to the network of the system. It also makes the detection system independent because it can convert it into a node that is part of the robot system in general. The whole system is implemented with Open Source software, based on the Ubuntu 14.04 LTS distribution.

\section{DETECTION OF VICTIMS}

The grayscale of the video camera is adjusted according to the radiation intensity received from the environment as well as the corresponding to the target and surrounding objects, it is analytically expressed as:

$$
W_{t o t}=\varepsilon \cdot \tau \cdot W_{o b j}+(1-\varepsilon) \cdot \tau \cdot W_{a m b}+(1-\tau) \cdot W_{a t m}\left(W / m^{2}\right)
$$

where $\varepsilon$ is the emissivity of the target, $\tau$ is the rate of transmission through the atmosphere, $W_{o b j}, W_{a m b}$ and $W_{a t m}$ is the energy or power emitted by each of the respective element. These energies are defined in the next expression:

$$
W_{x}=\varepsilon \sigma T_{x}^{4}\left(W / m^{2}\right)
$$

where the $\sigma$ is the Stefan-Boltzmann's constant $\left(5.67 \times 10^{-8}\right.$ $\mathrm{W} / \mathrm{m}^{2} \mathrm{~K}^{4}$ ) and $T_{x}$ is equivalent to $T_{a m b}$ that is the temperature of objects around the target, in other words the radiation they emit, and similar to $T_{a t m}$ that is the atmospheric temperature. This total radiation is scaled in 8 bits per pixel that provides the camera.

We integrate into the system a visual representation implementing personal false palette of colors, this allows monitoring and estimating the temperature of objects within the field of view (FOV). With this being explored the robot in hazardous areas or of difficult access, the operator, from the processing unit, can identify objects according to temperature, based on the surrounding temperature recorded by a thermometer in the search module. The contribution of this application is directing the camera to the points that likely show a victim by his temperature using free software, as shown in Fig. 4.

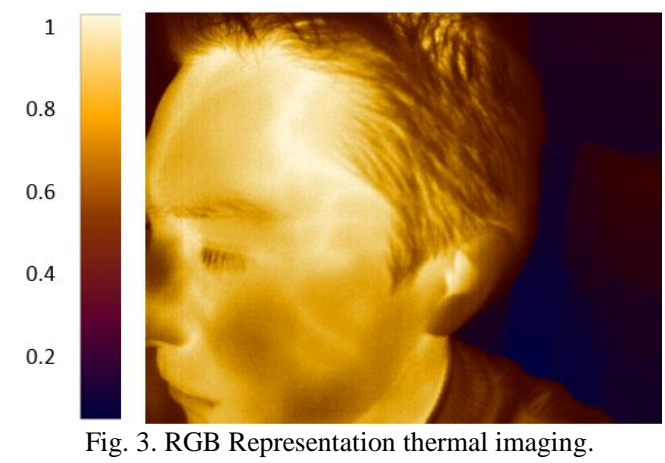

Based on thermal camera features and on different studies consulted and considered as basis, we propose the algorithm shown in Fig. 3 for unattended detection of non-surface victims, only if the system operator and the robot previously 
obtained the proposed points of interest.

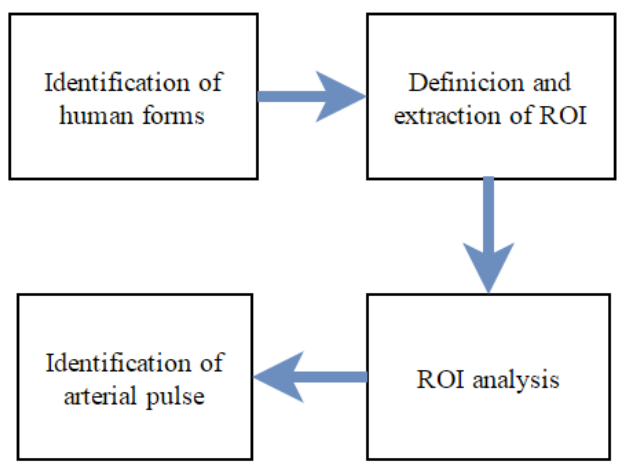

Fig. 4. Block diagram proposed for the detection of victim's algorithm.

\section{A. Identification of Human Forms}

We could define as search targets areas of the human body where you can measure the heart rate mainly arms, hands, head and neck for automatically search the region of interest (ROI). To make a detection regardless of any observer, we implement collections of Haar classifiers, for the full body, hands and head, training such classifiers with the OpenCV [8] tools.

If a positive detection is obtained, only that part of the video is displayed, and then we continue the definition and extraction of the region of interest, as shown in Fig. 4.

\section{B. Definition and Extraction of ROI}

The auto-configuration of the camera setting highlights the points with a greater temperature difference which are within the FOV, assigning the maximum values of the scale. Only if it's identified a body part that allows us to measure the pixels with the highest threshold which corresponds to the arteries and veins in the defined range of $100 \mathrm{mk}$ [5].

With the result of the classifiers, we extract the regions of the image that are candidates to be analyzed, a Gaussian filter is applied, with a core of $5 \times 5$ to soften the edges and then obtain a binary image according to interval threshold established by experimentation, defined with a length of 5 units, expressed as:

$$
M^{\prime}(\mathrm{i}, \mathrm{j})=\left\{\begin{array}{lr}
1 & T_{1} \leq M(i, j) \leq T_{2} \\
0 & \text { otherwise }
\end{array}\right.
$$

where $M(i, j)$ is the $320 \times 250$ array containing information of the video transmission, $M^{\prime}(j, k)$ is the binary image defined by the interval defined threshold $T_{1}$ and $T_{2}$. Subsequently, we have a number of regions $M^{\prime}(j, k)$, of which are only selected those that have areas larger than 100 pixels and less than 1500 , this range was established by experimentation. The areas within the range are defined by rectangular windows, finally they are analyzed by the non-maximum suppression algorithm to eliminate totally or partially those defined rectangular areas within other rectangular areas considering a factor of overlap of $65 \%$.

\section{ROI Analysis}

After determining the region of interest, we analyze it, because the amount of data is reduced, the same happens to the processing time. Our algorithm is divided into four stages, signal equalization, obtaining the signal, the filtering step and the detection step.

Equalization is performed so that the pixel values in each of the frames can be distributed throughout the range of possible values, which creates an image with visible contrasts. It consists of a transformation, where $P_{r}$ corresponds to the probability of occurrence of level $r_{k}, L$ is the number of levels which may represent a pixel, $n$ the total number of pixels of the image and $n_{k}$ the number of pixels with the value $r_{x} . S_{k}$ transformation is given by the equation, to transform the image simply map each $r_{k}$ level corresponding $s k$.

$$
\begin{gathered}
p_{r}\left(r_{k}\right)=\frac{n_{k}}{n} \quad k=0,1,2, \ldots, L-1 \\
S_{k}=T\left(r_{k}\right)=\sum_{j=0}^{k} p_{r}\left(r_{j}\right) \\
=\sum_{j=0}^{k} \frac{n_{j}}{n} \quad k=0,1,2, \ldots, L-1
\end{gathered}
$$

Once the image is equalized, the average value in each of the frames is obtained within the ROI, so that we obtain an arrangement of a dimension that describes the behavior of the ROI in the different frames, this will be our analysis signal.

For the filtering stage we employ the Continuous Wavelet Transform (CWT) where the image is decomposed into frequency and time. Later, we delete data that is not relevant and the signal with the inverse transform (ICWT) is recovered. CWT works with a mother $\left(\psi_{0}(\eta)\right)$ signal, in our case the Mexican hat, which allows us to have a good spatial resolution and can isolate the fine scales from the coarse ones, in our analysis small scales are of interest because it is where the heart rate variations occur. The main function is given by the equation and its graphic representation is shown in Fig. 5.
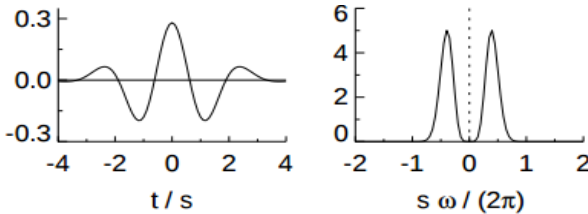

Fig. 5. Signal mother of the CWT

The WCT of a discrete sequence $x_{n}$ is defined as the convolution of $x_{n}$ with function $\psi_{0}(\eta)$ transferred and escalated.

$$
\Psi_{0}(\eta)=\frac{(-1)^{m+1}}{\sqrt{\Gamma\left(m+\frac{1}{2}\right)}} \frac{d^{m}}{d \eta^{m}}\left(e^{-\eta^{2} / 2}\right)
$$

Once we obtain the signal that models the behavior, we proceed to calculate the main frequency at which works, this frequency corresponds to the heartbeat.

\section{Identification of Arterial Pulse}

We have worked with $10 \times 10,30 \times 30,60 \times 60$ ROI of different window sizes and with different amounts of frames always multiple of $2 \frac{1}{2}$ in order to speed up the algorithms. To calculate the predominant frequency of the ROI we use the 
fast transformed Fourier (FFT), which is associated with the heart rate.

\section{RESULTS}

The results obtained so far have allowed us to obtain the signal that simulates the heartbeat, the algorithm was applied in different parts of the body with similar results, we can see in Fig. 6 a frame showing the full face and the location of the area where the artery predominates through the ROI.
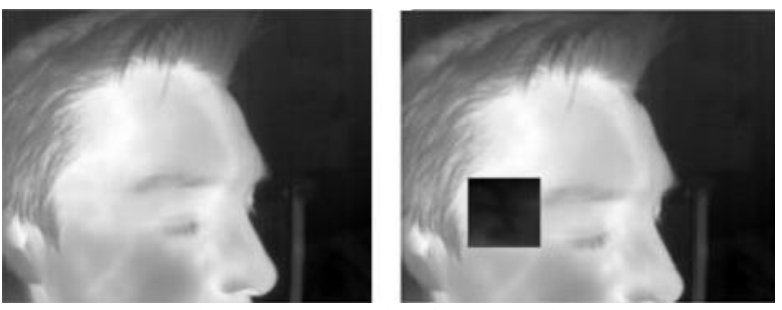

Fig. 6. ROI detection for processing.

In each of the frames the ROI average is extracted, and the $x$ signal is obtained, which is analyzed. In Fig. 7 we can see the graph of $x$, where the noise that occurs is deleted, to remove this noise and other unwanted frequencies, the CWT is used which is shown in the graph of Fig. 8.

The graphical representation of the CWT allows us appreciate the eye at first sight, in which the range of the image information is concentrated and so irrelevant information is discarded. In Fig. 8 (b) we can see the image of the CWT after selecting the data of interest.

Once filtering the signal, it is reconstructed by ICWT, we obtain a periodic signal or quasiperiodic signal, the FFT is applied to this signal to know its spectrum and determine its frequency.

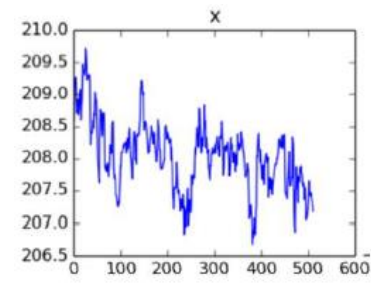

(a)

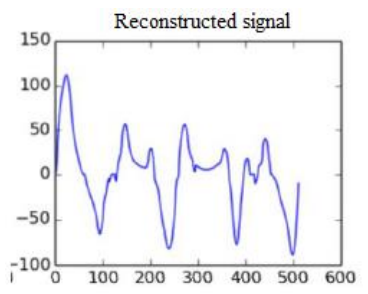

(b)
Fig. 7. (a) Graph of the variation of the average of the pixels in the ROI analysis time (b) graph of the average changes after applying CWT.

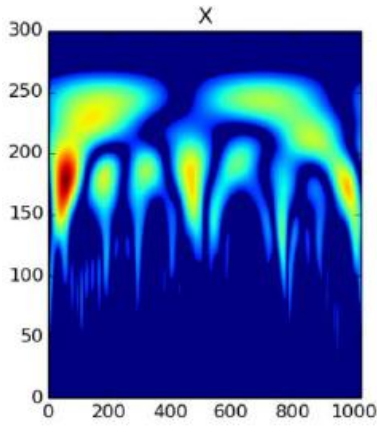

(a)

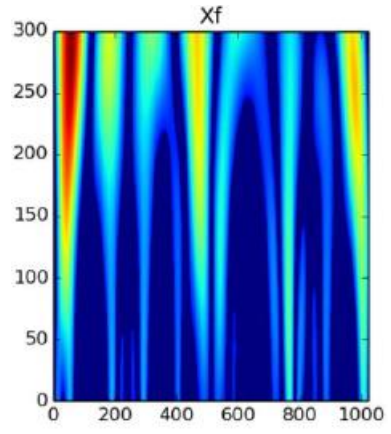

(b)
Fig. 8. (a) CWT representation of time and (b) representation of the frequency CWT.

The graphical representation of the CWT allows us appreciate the eye at first sight, in which the range of the image information is concentrated and so irrelevant information is discarded. In Fig. 8 (b) we can see the image of the CWT after selecting the data of interest.

Once filtering the signal, it is reconstructed by ICWT, we obtain a periodic signal or quasiperiodic signal, the FFT is applied to this signal to know its spectrum and determine its frequency (see Fig. 9).

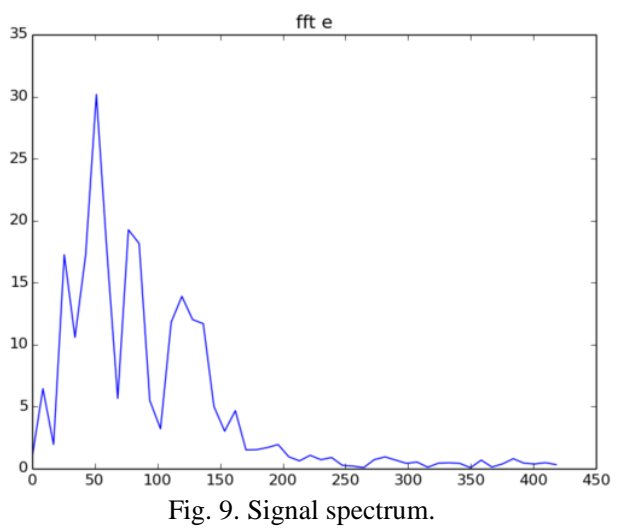

\section{CONCLUSIONS}

The Haar classifiers trained obtained acceptable results that detect the body parts of interest, regardless of an observer to find regions of interest to apply pulse detection. It will work on other proposed algorithm to find the anthropomorphic shapes that reduce the use of processor and memory to apply it in real time.

Regarding the identification of the pulse, the results differed six-pulse compared to the measurement recorded at the time of acquisition of the image bank, the tests were performed with three people. The next stage of this work corresponds testing with different people and in real environments. The proposal of this work protects rescuers search for victims of exposure to dangerous places even for dogs, optimizing search time using robots.

\section{ACKNOWLEDGMENT}

This research is conducted through the Program UNAM DGAPA PAPIIT IT102615 "Unconventional robots for browsing and searching tasks".

\section{REFERENCES}

[1] S. Tadokoro, Rescue Robotics, DDT Project on Robots and Systems for Urban Search and Rescue, 2009, Springer.

[2] S. Y. Chekmenev et al., Multiresolution Approach for Noncontact Measurements of Arterial Press Using Thermal Imaging, Augmented Vision Perception in Infrared, Springer London, pp. 87-112, 2009.

[3] B. Guha et al., "Detecting press from head motions in video," Computer Vision Foundation, pp. 3430-3437, 2013.

[4] J. Fei and I. Pavlidis, "Thermistor at a distance: Unobtrusive measurement of breathing," IEEE Transactions on Biomedical Engineering, vol. 57, no. 4, April 2010.

[5] T. Togawa, "Non-contact skin emissivity: Measurement from reflectance using step change in ambient radiation temperature," Clin. Phys. Physiol. Meas, vol. 10, pp. 39-48, 1989.

[6] M. Yang, Q. Liu, T. Turner, and Y. Wu, "Vital sign estimation from passive thermal video," in Proc. the 2008 IEEE Conference on Computer Vision and Pattern Recognition, 2008, pp. 1-8.

[7] J. Lentin, Mastering ROS for Robotics Programming; Packt Publishing Ltd., UK, December 2015. 
[8] H. Joseph, Open CV Computer Vision with Python, Packt Publishing Ltd, UK, December 2014.

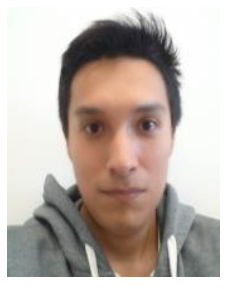

Carlos I. García S. was born in Mexico City, Mexico, in 1990. He received the B.S degree in electrical and electronic from the National Autonomous University of Mexico (UNAM), Mexico city, Mexico, in 2016.

In 2009, he was in admission to the club of robotics engineering faculty, where he has participated in national and international competitions of robotics. With the project of robot cleaner beach, participated and won fourth place in 2013 LARC Peru, the first in the TMR 2014, Mexico and the second in the international competition of robots beach in Greece. Then he works in the project led by the M.I Yukihiro Minami in the Faculty of Engineering at UNAM and is currently studying the master grade in digital signal processing.

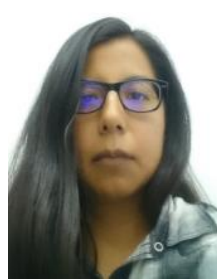

A. Yoloxóchil J. R. was born in Mexico City, México, 1980. She received the B.S degree in electrical and electronic engineering from the National Autonomous University of Mexico (UNAM), Mexico city, Mexico, in 2010 .

In 2008, she joined the Division of Basic Sciences at UNAM as an assistant professor; in 2011 as an subject teacher.

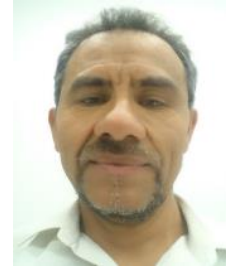

Larry H. Escobar was born in Guatemala, Central America, in 1961. He received the B.S degree in mechanical and electrical engineering and the M.S degree in electrical engineering from the National Autonomous University of Mexico (UNAM), Mexico city, Mexico, in 1992 and 1997, respectively.

In 1991, he joined the Department of Electrical Engineering at UNAM as an assistant professor, in 1992 as an associate professor, and in 1994 he became

a full-time professor.

His current research interests include digital filtering, spatial filtering and real-time digital signal processing. Currently, he is coordinator of the Department of Digital Signal Processing, Faculty of Engineering, UNAM. $\mathrm{He}$ has published about 45 papers about his technical area in congresses, magazines and books. He has directed 20 undergraduate engineering theses and over 10 postgraduate engineering theses. During his academic work, Prof. Escobar has received tree special cathedra awards from the Faculty of Engineering, UNAM. 